\title{
Endovascular iliac and femoral angioplasty followed by contralateral femoropopliteal bypass in a patient with chronic lower extremity ischemia: case report
}

\begin{abstract}
Hybrid procedures allow complete revascularization of peripheral arteries in patients with complex multilevel arterial disease. Here, we present a 58 -year-old male patient with intermittent claudication of both legs. Total occlusion of the right iliac artery, common femoral artery and superficial femoral artery were traversed using a guidewire retrogradely through the left superficial femoral artery. Drug eluting balloon was followed by stent implantation to the external iliac artery. Once patency of the vessel was seen on the angiogram, femoropopliteal bypass had to be performed on the left leg as the patient had developed pain and pallor of the left leg. Angioplasty of the left side during the same session was avoided as retrograde approach through the right superficial femoral artery could jeopardize the stent and cause migration.
\end{abstract}

Keywords: Endovascular intervention, percutaneous transluminal angioplasty, femoro-popliteal bypass, limb ischemia, iliac stent, surgical techniques, hybrid procedures, vascular diseases, acute leg pain, pallor, contralateral leg, ipsilateral extremity
Volume 6 Issue 6 - 2018

\section{Görkem Yiğit, Mehmet Cahit Sarıcaoğlu,Anıl Özen, Hakkı Zafer İșcan \\ Turkiye Yuksek Ihtisas Training and Research Hospital,Turkey}

Correspondence: Görkem Yiğit, Altınkuleler Sitesi, Altındağ Sakarya mahallesi, Yönü Sokak 06080 Ankara/ Turkey, Tel +90 50667775 77, Email drgorkemyigit@gmail.com

\section{Received: November 24, 2018 | Published: December 27,} 2018
Abbreviations: DEB, drug eluting balloon; ATA, anterior tibial artery; PTA, and posterior tibial artery; DSA, digital subtraction angiography; SFA, superficial femoral arteries; EIA, external iliac arteries; CIA, common iliac arteries; TASC, trans-atlantic intersociety consensus

\section{Introduction}

Hybrid vascular procedures are methods that combine both open surgical techniques and endovascular interventions in the management of vascular diseases. Endovascular treatment may be used for in-flow or out-flow lesions in combination with open surgery. ${ }^{1,2}$ The first reported hybrid vascular surgical procedure reported in the literature dates back to 1973. Porter and colleagues performed dilatation of the pelvic arteries alongside femoro-femoral bypass. ${ }^{3}$ Combining two types of approaches in the peripheral vascular field is a less aggressive alternative and possess results that are comparable to the conventional treatment. ${ }^{4}$ Furthermore, hybrid procedures shorten the duration of the surgical procedures. ${ }^{5}$ Currently, hybrid procedures such as combined femoral endarterectomy and iliac angioplasty/stenting, combined iliac artery stenting and distal bypass graft, simultaneous SFA endovascular intervention and popliteal to distal bypass, ipsilateral iliac stent and femoro-popliteal bypass procedures have been documented to consist of 5 to $21 \%$ of the total number of vascular reconstructions. ${ }^{6}$ However, hybrid procedures are mostly performed in the ipsilateral extremity; the number of cases that require interventions to both lower extremities simultaneously remain scarce. Here, we present a patient in whom occluded vessels of the right lower extremity were treated with drug eluting balloon (DEB) and stent implantation. A femoropopliteal bypass had to be performed on the contralateral leg as the patient developed acute leg pain and pallor.

\section{Case report}

A 58-year-old male patient was admitted to our clinic with a complaint of pain in both legs with intermittent claudication. He had a medical history of 25-years of smoking, diabetes mellitus and hypertension. On physical examination, anterior tibial artery (ATA) and posterior tibial artery (PTA) pulses were non-palpable on both sides. The patient had Fontaine stage $2 \mathrm{~b}$ symptoms according to the classification of peripheral arterial diseases. Digital subtraction angiography (DSA) revealed that both superficial femoral arteries (SFA), external iliac arteries (EIA) and common iliac arteries (CIA) were totally occluded (Figure 1-3).

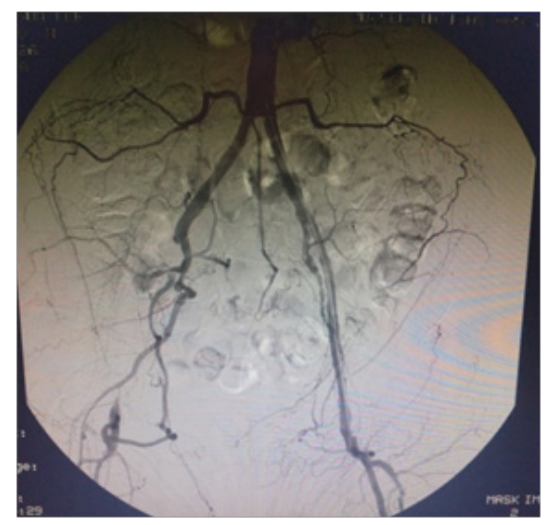

Figure I DSA showing occlusion of the common iliac arteries

Since the patient's claudication was more pronounced in the right leg, a decision to proceed with interventional treatment of this leg was made. The procedure was performed in the hybrid operation room under local anaethesia. The Seldinger technique was used for imaging and sheath was placed into the left femoral artery guided retrogradely to the right side Figure 4. The total occlusion of the right iliac artery, CFA and the SFA were passed by the guidewire and DEB was performed (Figure 4-7). 


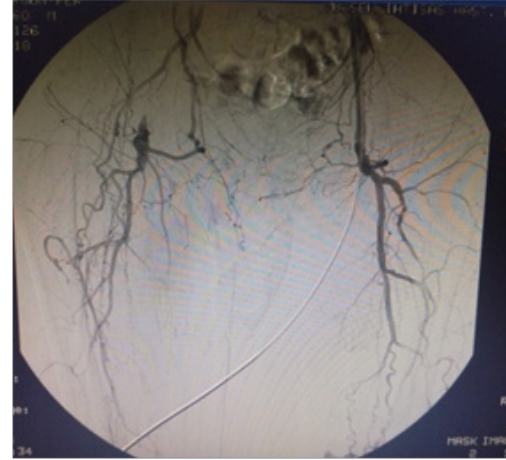

Figure 2 Occlusion of the common femoral arteries

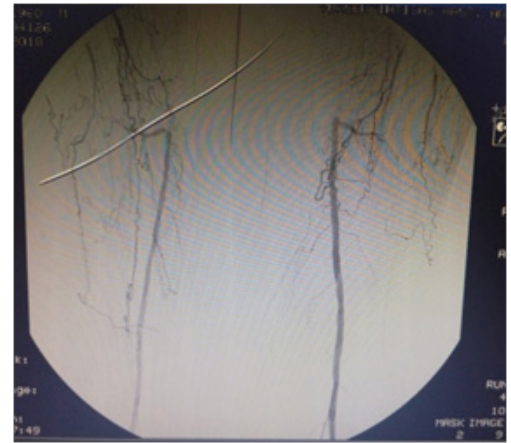

Figure 3 Occlusion of the superficial femoral arteries

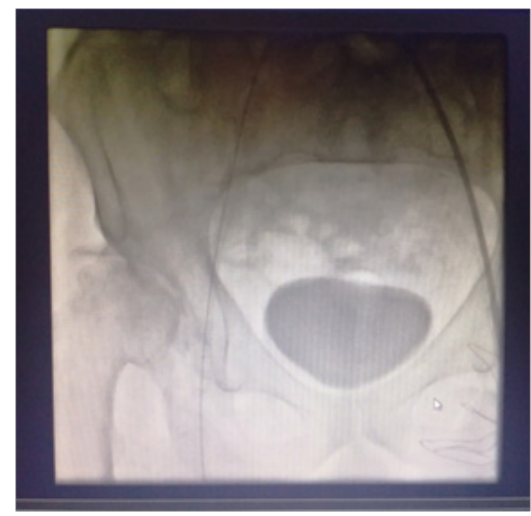

Figure 4 Femoral artery guided retrogradely

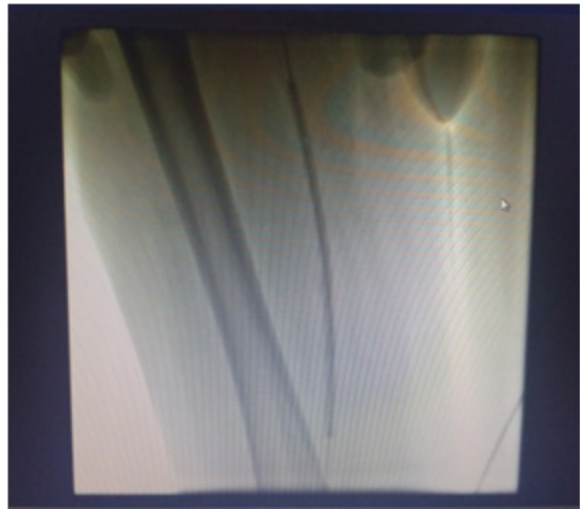

Figure 5 DEB was performed superficial femoral artery

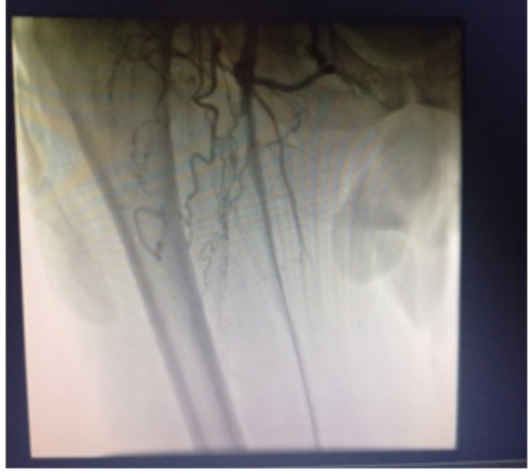

Figure 6 After DEB opened superficial femoral artery

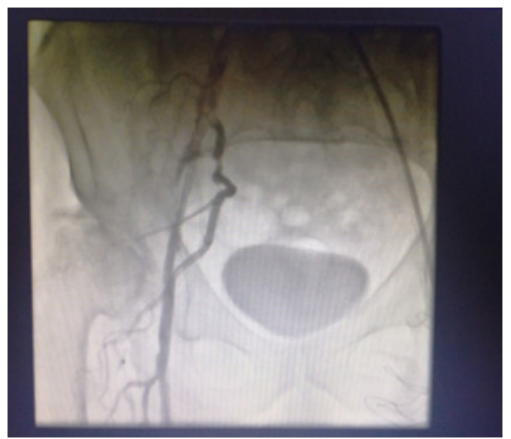

Figure 7 DEB was performed common iliac artery

Due to the very tight stenosis and long occlusive segment of the iliac, a stent was placed at the site Figure 8. After the stenting procedure, angioplasty was applied to the residual stenosis Figure 9. An hour after patency of the right sided vessels had been achieved, the patient presented with pallor and pain of the contralateral leg. DSA of the left leg did not display any evidence of an embolus or proximal occlusion. The patient had already been heparinized. Nevertheless, the symptoms did not improve. Angioplasty of the left side via the right SFA was avoided since a retrograde approach could jeopardize the stent and cause migration during the same session (Figure 8-12).

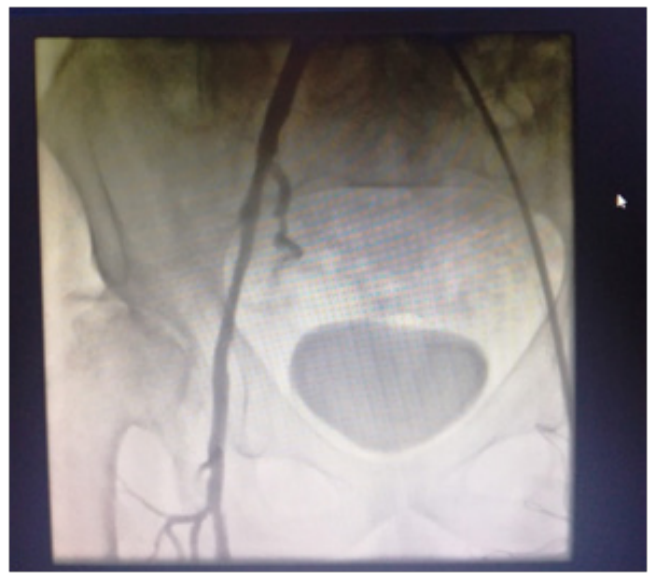

Figure 8 Stent was placed at the site

Hence, a decision to perform femoropopliteal bypass of the left leg under general anaesthesia was made to which the patient consented. An additional dose of heparin was administered prior to clamping of 
the femoral vessels. Femoropopliteal bypass was performed using an 8 $\mathrm{mm}$ polytetrofluoroethylene graft after which the patient's symptoms resolved. He was discharged from the hospital on the 3 rd postoperative day. The patient was followed up with $75 \mathrm{mg}$ daily clopidogrel and $100 \mathrm{mg}$ acetylsalicylic acid. At the two month postoperative followup, the patient's walking distance had increased, no complications of surgery were detected, and bilateral pedal pulses were present.

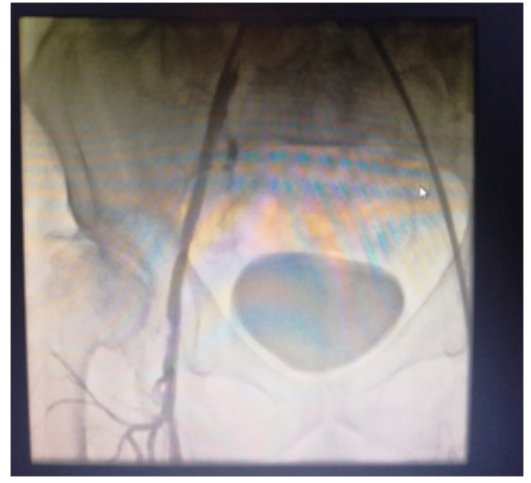

Figure 9 Angioplasty applied to the residual stenosis

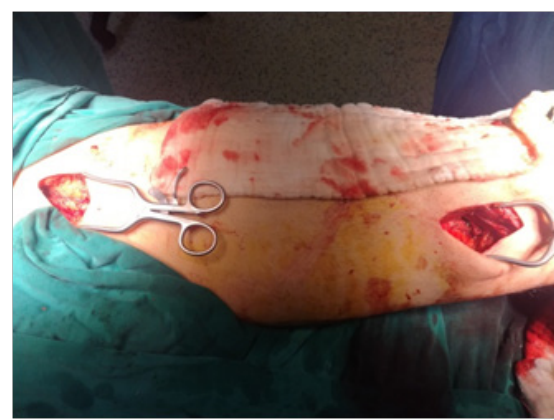

Figure 10 Femoro-popliteal bypass performed left leg

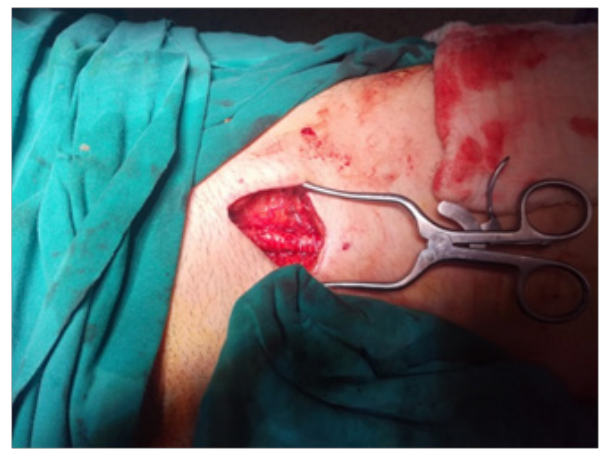

Figure I I Femoral side of anostomosis with PTFE graft

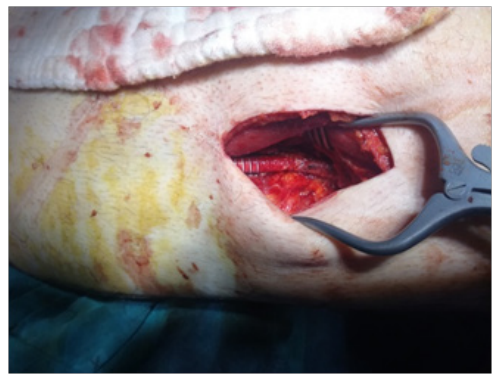

Figure I 2 Popliteal side of anostomosis with PTFE graft

\section{Discussion}

Hybrid techniques can safely be performed in the vascular operating room providing that the inventory is equipped for endovascular interventions. Especially in the last decade, vascular surgeons have become increasingly interested in and experienced in the field of percutaneous transluminal angioplasty and stenting procedures. They should be able to combine endovascular procedures with surgical ones in a creative fashion with a prompt decision to the benefit of their patients. Several studies have demonstrated that the ideal treatment for patients with multilevel disease is proximal angioplasty or stenting combined with distal infrainguinal reconstruction. ${ }^{7}$ Piazza ${ }^{1}$ performed a retrospective study in which data on the outcomes of 75 patients (76 limbs) treated with concomitant iliac stenting and femoropopliteal bypass was presented with good outcomes. Similarly, Liu $\mathrm{C}^{8}$ published a paper on their experience in iliac angioplasty and stenting combined with simultaneous femoro-popliteal bypass on 12 lower extremities of 10 patients suffering from multilevel atherosclerotic occlusive disease. Furthermore, the study of Ahmed Mousa ${ }^{9}$ included 10 patients treated with a combined procedure with excellent results and Brewster ${ }^{10}$ reported good long-term results with combined iliac angioplasty and distal arterial reconstruction. In contrast to this, we had to perform contralateral femoro-popliteal bypass after iliac stenting of the right side. The Trans-Atlantic Inter-Society Consensus (TASC) classification suggests the choices for first-line therapy and predicts successful intervention after endovascular or open surgical therapy mainly according to the site of the lesion and its length. ${ }^{11}$ The effect of factors such as patient comorbidity, patient choice and the experience of the patient on decision making is emphasized. In this case, the patient had a TASC-D lesion however; endovascular revascularization of the right leg was opted for as the patient did not consent to open surgery. In spite of this, shortly after the initial intervention has been completed, an emergency decision was made to perform femeropopliteal bypass, an open surgical procedure, of the contralateral leg due to the development of pallor and pain on that side. Approaching via the right SFA was avoided as taking a retrograde approach during the same session can jeopardize the stent and cause migration.

\section{Conclusion}

Especially in the last decade, vascular surgeons have become increasingly interested in and experienced in the field of percutaneous transluminal angioplasty and stenting procedures. We have been performing angioplasty and drug eluting balloons, as cardiovascular surgeons, for peripheral arterial diseases in our clinic for 4 years. This is an atypical case that we encountered since an endovascular approach to the right lower extremity was planned. However, we had to perform femoro-popliteal bypass to the contralateral leg due to the patient developing acute symptoms. We also tried to emphasize the importance of cardiovascular surgeons combining endovascular procedures with surgical ones where necessary.

The benefits of simultaneous endovascular and open treatment ${ }^{12}$

a) No delay in complete revascularization of the ischemic limb

b) Open surgery can immediately repair inadequate endovascular results and vice versa

c) Puncture complications related to angioplasty are eliminated

d) Potential infectious complications of two separate interventions are minimized 
e) Adjustment of drug therapy between the procedures is not required

f) Hospital stay is shortened and possibly cheaper

g) Due to possible routine use of angiography and open flow measurements at the end of the intervention, surgeons have better means to control and verify renewed blood flow at all levels and lesion sites.

\section{Acknowledgments}

None.

\section{Conflicts of interest}

The authors declare that there is no conflicts of interest.

\section{References}

1. Piazza M, Squizzato F, Lepidi S, et al. Iliac artery stenting combined with ipsilateral open femoro-popliteal revascularization and its effect on bypass patency. Ann Vasc Surgery. 2017;44:282-288.

2. Lantis J, Jensen M, Benvenisty A, et al. Outcomes of combined superficial femoral endovascular revascularization and popliteal to distal bypass for patients with tissue loss. Ann Vasc Surg. 2008;22(3):366-371.

3. Porter JM, Eidemiller LR, Dotter CT, et al. Combined arterial dilatation and femorofemoral bypass for limb salvage. Surg Gynecol Obstet. 1973;137(3):409-412.

4. Parodi C, Ferreira L. Combined Surgical and Endovascular Approaches. 2nd Virtual Congress of Cardiology. 2001.

5. Schrijver AM, Moll FLFL, De Vries JP. Hybrid procedures for peripheral obstructive disease. J Cardiovasc Surg. 2010;51(6):833-843.
6. Ebaugh JL, Gagnon D, Owens CD, et al. Comparison of costs of staged versus simultaneous lower extremity arterial hybrid procedures. Am J Surg. 2008;196(5):634-640.

7. Aboyans V, Ricco JB, Bartelink MEL, et al. 2017 ESC Guidelines on the Diagnosis and Treatment of Peripheral Arterial Diseases, in collaboration with the European Society for Vascular Surgery (ESVS): Document covering atherosclerotic disease of extracranial carotid and vertebral, mesenteric, renal, upper and lower extremity arteries Endorsed by: the European Stroke Organization (ESO) The Task Force for the Diagnosis and Treatment of Peripheral Arterial Diseases of the European Society of Cardiology (ESC) and of the European Society for Vascular Surgery (ESVS). Eur Heart J. 2018;39(9):763-816.

8. Liu C, Guan H, Li Y, et al. Combined intraoperative iliac artery stents and femoro-popliteal bypass for multilevel atherosclerotic occlusive disease. Chin Med Sci J. 2001;16(3):165-168.

9. Mousa A, Abdel-Hamid M, Ewida A, et al. Combined percutaneous endovascular iliac angioplasty and infrainguinal surgical revascularization for chronic lower extremity ischemia: preliminary result. Vascular.2010;18(2):71-76.

10. Brewster DC, Cambria RP, Darling RC, et al. Long-term results of combined iliac balloon angioplasty and distal surgical revascularization. Ann Surg. 1989;210(3):324-331.

11. Lyden SP, Smouse HB. TASC II and the endovascular management of infrainguinal disease. $J$ Endovasc Ther. 2009;16(2 Suppl 2):II5-II18.

12. Balaz P, Rokosny S, Bafrnec J, et al. The role of hybrid procedures in the management of peripheral vascular disease. Scand $J$ Surg. 2012;101(4):232-237. 